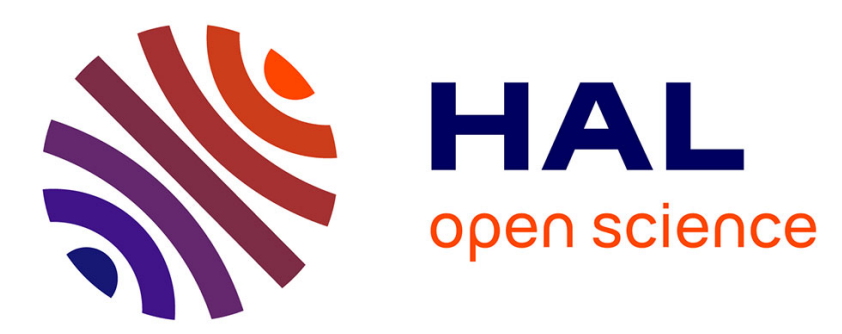

\title{
Agent-Based Facilitation of Water Allocation: Case Study in the Drome River Valley
}

Olivier Barreteau, Patrice Garin, Alexandre Dumontier, Geraldine Abrami, Flavie Cernesson

\section{- To cite this version:}

Olivier Barreteau, Patrice Garin, Alexandre Dumontier, Geraldine Abrami, Flavie Cernesson. AgentBased Facilitation of Water Allocation: Case Study in the Drome River Valley. Group Decision and Negotiation, 2003, 12 (5), pp.441 - 461. 10.1023/B:GRUP.0000003743.65698.78 . hal-01575702

\author{
HAL Id: hal-01575702 \\ https://hal.science/hal-01575702
}

Submitted on 19 Nov 2019

HAL is a multi-disciplinary open access archive for the deposit and dissemination of scientific research documents, whether they are published or not. The documents may come from teaching and research institutions in France or abroad, or from public or private research centers.
L'archive ouverte pluridisciplinaire HAL, est destinée au dépôt et à la diffusion de documents scientifiques de niveau recherche, publiés ou non, émanant des établissements d'enseignement et de recherche français ou étrangers, des laboratoires publics ou privés. 


\section{Agent-Based Facilitation of Water Allocation: Case Study in the Drome River Valley}

OLIVIER BARRETEAU, PATRICE GARIN, ALEXANDRE DUMONTIER, GÉRALDINE ABRAMI

Cemagref, Irrigation Unit, BP 5095, F-34033 Montpellier, France (E-mail: olivier.barreteau@cemagref.fr)

FLAVIE CERNESSON

UMR 3 S Cemagref/ENGREF, BP 5035, F-34033 Montpellier, France (E-mail: flavie.cernesson@teledetection.fr)

\section{Introduction}

As a negotiation and group decision support system, computer tools have empirically proven to be worthwhile in increasing joint outcomes in dyadic negotiations (Lim 2000) or in assisting in international negotiations (Druckman, Harris, and Ramberg 2002). Among these, use of simulation tools and notably Agent-Based Simulations is supposedly well underway. Their use is suggested with increasing frequency in recent literature because of their ability to explain the dynamics of complex systems (Bousquet et al. 2002; Doran 1999; Moss and Davidsson 2001). In this paper, we will illustrate, by way of a field experiment, how a negotiation process involving water allocation among farmers in a river basin is fostered by the use of Agent-Based Models. This is tested through their involvement in a collective decision process with new collective rules for water allocation in the South of France. By supporting a specific collective decision process we are able to compare two successive 
computer models designed for that purpose: one that is Macro $^{\circledast}$ Excel $^{\circledR}$, called SimSage, and one that is Agent based, called GibiDrome.

This kind of use of Agent-Based Modeling as a negotiation or collective decision support tool is based on a difference in scales used in the model and the negotiation process: time steps and behavioral patterns in particular are at finer grain than the decision process they are designed to support. This use of ABM as models for negotiation breaks from the more frequent use of MAS as a model of negotiation. While modeling of negotiation seeks an isomorphism between model and negotiation process through a focus on analysis and representation of the agent's rationality and communication protocols, modeling for negotiation seeks to provide information about the system concerned in the negotiation. Here, the purpose of GibiDrome is to provide information on potential consequences of outcomes of discussion on the whole system dynamics.

The case study and the two successive models are fully described. Both are compared, with special attention given to their adaptability and the kind of information they provide. This comparison tackles the way they interfere with the negotiation process in particular.

\section{Models to support negotiation}

If models of negotiation are increasingly developed, they may not be sufficient to support negotiation, since they will broaden participants' information from a specific point of view only. The use of models within the collective decision process is also at stake, at least as regards collective decision protocols. A key point is that these models need not be at the same organizational level as the negotiation they are designed to support.

\subsection{From models of negotiation towards models for negotiation}

The main trend is to simulate negotiation on the basis of an agent-based model. Both modeling and negotiation science involve either modeling or simulation results.

From a computer science standpoint, Multi-Agent Systems may need the enforcement of conflict resolution among computer agents, regardless of what they may represent. The purpose here is to learn from negotiation science in order to design conflict resolution algorithms (Faratin et al. 2001; Grosz 2000). Models of negotiation are then used as a paradigm to represent these algorithms. They are assessed based on their capacity to solve conflicts, and not on their validity as a negotiation model.

From a negotiation science point of view however, the purpose is to take advantage of the power of MAS to simulate discussions and negotiations in order to reach a better understanding of consequences of specific theoretical models or protocols of negotiation patterns. Theorists will check biases induced by their models, whereas practitioners or extension officers might use these simulations to set up a context favorable to a conclusion of a negotiation. In both cases, negotiation is done on the same organizational scale as the model, since an isomorphism is being sought. Negotiation is internalized in the model. MAS is used concurrently with other tools such as game theory models, expert knowledge, or 
role-playing games. It might be assessed through either theoretical progress or in comparison with other concurrent tools having the same objectives, as in (Green 2002). Their purpose is to simulate and possibly forecast outcomes of negotiations, whether they be real or virtual.

As far as mediation and negotiation support are concerned, such internalization of negotiation in the model requires giving stakeholders participating in the negotiation a model that represents their involvement in this process. This kind of negotiation support is used for complex institutional patterns in which stakes are either not too complex or not looked at thoroughly. This is the case, for example, when the existence of an outcome, more than the nature of this outcome, is an indicator of success in itself. Negotiation based on principles of management and definition of clusters is an example (Burkardt, Lamb, and Taylor 1998). It is assumed in these cases that stakeholders wish to reach an agreement and that the negotiation pattern itself may help them reach this agreement. For (Druckman, Harris, and Ramberg 2002), the simulation of dynamics and outcome of an on-going negotiation requires that actors in that negotiation adapt strategically in order to improve the outcome, or even to reach one. All these cases assume that the first issue, that of getting stakeholders to negotiations, has already been resolved, which is a big assumption in many cases.

Another way to broaden the field of information available to participants (Benbasat and Lim 2000) is to give them more insight into the processes at stake. Providing stakeholders with potential consequences of various choices involved in an on-going group decision process reportedly mobilizes them more actively in the process (Driessen, Glasbergen, and Verdaas 2001), which is a way to alleviate the aforementioned problem. Simulation at this point provides a better understanding of a complex system and of its heterogeneous points of view. The model is used to simulate dynamics of the actors' relations to stakes in the negotiation. This may involve simulation of negotiation but at a different organizational level: it may be interesting to note that a model designed to support a collective decision involving a constitutional or macro time scale simulates negotiations as regards the operational or micro time scale. Whereas, in the previous paragraph, negotiation is included in the model, here the model is included in negotiation.

This paper deals only with this last kind of relationship between model and negotiation, and shows the relevance of MAS for that use. It is based on an action research experiment in the South of France that deals with negotiation involving water allocation rules. Based on this case study, this paper will explore the paths through which that use of MAS might provide support to negotiation and collective decision processes. This is dependent on a specific framework for the group decision process, the patrimonial approach, which will be described first.

\subsection{Description and organization of negotiation process}

Literature provides many approaches to describing and organizing a negotiation process from the facilitator's point of view:

- the "sequential approach" divides the entire negotiation process into stages such as identification of participants, information, discussion, alternative proposition, co-operation, 
agreement. Each stage has its own role and importance and should be tackled individually, but in real negotiations such separation between stages may appear rather theoretical;

- the "typological approach" provides two main families of negotiation: conflicting or cooperative. These constitute two theoretical references: real negotiations that are really a combination of both;

- the "actors' interactions approach" tries to build on relations among actors, such as power relations, to understand the process. Negotiations are then described through modes of interaction among negotiators;

- the "decision process approach" states that negotiation is only a means to progress towards a decision, at given scales of duration and organization, which leads to several kinds of negotiation: interstitial, component or global (Faure et al. 1998).

This diversity in description and analysis also leads to various approaches to facilitation of negotiations. We opt for following a "patrimonial approach" based on the principle of defining the desired future rather than trying to forecast it. This pattern has been chosen because it is well suited to the whole pattern of collective water management in France. This "patrimonial approach" is organized around the following five stages (Babin and Bertrand 1998; Weber 1996, 1998):

1. sharing representations of the system and agreement on the stakes of the negotiation,

2. agreement on a long-term objective (building "constitutional choices"),

3. suggestions for scenarios by stakeholders involved in the process, including operational rules for its implementation and assessment, in order to reach this long-term objective,

4. discussion on scenarios with the long-term objective in mind and agreement on one of them,

5. legitimizing and ritualizing this scenario.

The second stage may also include a ritualisation step in order to anchor the agreement on the long-term objective at a constitutional level. Each stage except the third and fifth are negotiations in themselves: debate on the current situation and trends in the first stage, agreement on the long-term objective in the second, and choice of the scenario of collective rules in the third. However, as a whole process it is quite a large time scale negotiation, since it is meant to first tackle constitutional choices constraining operational ones, if we follow Ostrom's terminology (Ostrom 1990).

This approach focuses on co-operative negotiation, with the major assumption that an agreement might be reached on the long-term objective. Here "patrimonial" refers to the kind of world stakeholders involved in the collective decision process wish to pass on to their children. However, the entire negotiation process does not deal with this issue of legacy nor with the choice of a long-term objective, but through discussion over such a long time period, it is assumed that negotiators learn by talking to each other, and that trust among them is increasing. This facilitates and frames then the discussion on shorter-term features and notably operational rules.

This approach belongs to the family of post-normal science (Funtowicz et al. 1999): like other authors who find themselves in such approaches we submit that the collective deci- 
sion process itself is more important than the quality of its outcome. These approaches are based mainly on the statement that the evolution of complex systems depends on many random parameters over which players have no control. Therefore a management organization that is adaptive and participatory in nature will better deal with this uncertainty and incorporate some of the uncontrollable parameters. An optimal solution, if any exists, would be transient. We assume then that the five stages of patrimonial approach raise the perception of the process's quality over the long term due to an increase of its legitimacy.

Use of models in this context is suitable at least for stages 1 and 4 . In stage 1 , it is the modeling process itself that is relevant. A discussion of the model's assumptions in a collective setting leads participants to make their own worldviews explicit and discuss them collectively. In stage 4, it is the simulations that are most worthwhile in providing information on possible consequences of scenarios being tested according to the model's assumptions. Stage 3 may also benefit from the use of models. Scenarios proposed by the stakeholder are easier to understand when they can de described dynamically. Simulation models might provide support for that. From this point on, we will focus on the collective decision processes that are organized this way.

\subsection{Specific needs in cases of complex systems}

As far as complex systems are concerned, as regards water allocation issues, modeling is supposed to improve and aid in sharing information among stakeholders (Simonovic 2000). This is linked with two different uses of models: in group session or in individual sessions. In a group setting, the model is supposed to foster discussions and improve sharing of information. In an individual setting, the model is supposed to provide information to stakeholders, focusing more on their personal criteria. In the discussion that follows, only the first has been tested on a limited scale, and is presented in Section 3.

A first requirement, already commonly acknowledged, is cross-disciplinarity. Even if the negotiation process itself is not at stake, the dynamics of the system under discussion, even for a short time scale, involves social as well physical processes.

Other requirements deserve more discussion. Interactions among stakeholders as regards natural resource management may take several channels: indirect, through the perception of impact on the resource caused by others' actions, or direct, either on a one on one basis or in institutionalized collective frameworks (Rouchier et al. 1998). All these direct interactions are based on mediating objects. They are discussion support tools. They can act through the constitution of a shared representation or the simulation of proposed scenarios of collective rules. They make an additional channel of interaction available among stakeholders: interaction on the intermediary object. This interaction can be exchanged through evolving versions that are passed on from one to another or shared in a collective setting. Dead-end conflicts caused by misunderstandings may thus be prevented, provided that it is perceived as a legitimate mediating object. An Agent-Based Model constitutes just such a mediating object, inducing stakeholders to agree on or at least discuss a common representation of their joint natural resource system, facilitating their communications regarding it. 
With the use of simulation models as discussion support tools comes the possibility of making common knowledge problems encountered by every stakeholder on its own, which are used to be diluted in time and space (Barreteau, Bousquet, and Attonaty 2001). Misrepresentations or bad faith behaviors can thus be prevented. The use of a simulation tool leads to a concentration of time and space of selected processes. The evolution of each stakeholder's representations is strengthened, providing each one with better knowledge of the others' representations. Shared representations mediate this evolution towards improved cognitive capacities of stakeholders (Teulier-Bourgine 1997). So the legitimacy of the decision process is increased through a better knowledge of its foundations and construction, rather than of a supposed optimality (Funtowicz et al. 1999). Therefore, the most immediate need of models used as group decision support with complex systems is the legitimacy of their use in the decision process where specific stakes are concerned.

Post-normal science focuses on the uncertainty and evolutionary nature of open complex systems. Since modeling also has its own pace, the artifacts produced evolve as well, to keep in step with issues at stake and representations with participants of a collective decision process throughout this process. The use of simulation tools in a post-normal approach is an interaction between two processes, modeling on one hand and collective decision on the other. There is no better model than the one evolving with the collective decision process. This is the second major need for tools used in a negotiation support setting.

The first results on the suitability of MAS as a mediating object in discussions have already been published (Bousquet et al. 1999, 2002). In this paper, their use in a real negotiation process is described and compared to another more classical modeling approach.

\section{An experimental case study in the Drome River Valley}

Authors are currently involved in an action research project that supports a water basin institution of the kind established by the French Water Act, for the purpose of designing suitable negotiation support tools and their methodology of use. This section describes the context of the negotiation, two models that have already been developed, and the protocol for their use.

\subsection{Towards collective rules for sharing water during dry season}

The 1992 French Water Act has defined a framework for local water institutions: SAGE and CLE. SAGE stands for "schéma d'aménagement et de gestion des eaux" ("water management and operation plans"). CLE stands for "commission locale de l'eau" ("local water commission"). SAGE is a five-year water management plan on which stakeholders have agreed within CLE, and which the State's local representative, the prefect, has signed. The 1992 act defines the composition of CLE as follows:

- $25 \%$ representatives of state institutions,

- $50 \%$ local elected people,

- $25 \%$ representatives for users. 
It is responsible for the design and enforcement of SAGE.

Territorial divisions are decided by upper level water institution ${ }^{1}$ based on hydro-geographical boundaries, such as a sub-watershed or an aquifer. Creation of a SAGE is not required, but should be based on a local initiative. If a SAGE is formed, the above framework is compulsory.

SAGE documents describe the future status of the hydrosystem on which stakeholders have agreed. This includes the objectives stated for the hydrosystem by stakeholders. It is the responsibility of CLE to mediate the negotiation of scenarios to reach and to enforce them. Therefore the whole process may fit the organizational framework of the negotiation process described above, that of a patrimonial approach:

- stage 1 is an initialization with identification of concerned parties and debate on current status and trends of the hydrosystem,

- stage 2 corresponds to the creation of SAGE,

- stage 3 is the responsibility of CLE whose composition favors real discussions on scenarios,

- stage 4 should emerge from stage 3 through mediation of CLE,

- stage 5, ritualizing, in French secular society is the signing by the prefect, ${ }^{2}$

It is therefore relevant to search for negotiation support tools fitting this negotiation framework. We are now just after the first ritualisation of the long-term objective by the prefect. However, an important limit in the identification of the SAGE process in a patrimonial approach is linked to the definition of "long-term". SAGE is valid for 5-10 years, which is shorter than the long-term scale in a patrimonial approach (25-30 years). Nevertheless, we will maintain this representation of the SAGE process as a patrimonial approach, not only because it provides a useful framework for analysis in the use of models, but also because these objectives, which are currently mid-term, refer to longer-term principles such as typology of occupation in the territory. In the Drome river valley, the issue deals explicitly with an agricultural area on the one hand and an area that is primarily tourist-oriented on the other.

The SAGE of the Drôme River Valley was the first one signed at the end of 1997. Moreover, it has a relatively high-level content and is quite ambitious. Issues at stake are:

- gravel extraction management,

- minimum flows to be observed in downstream part,

- clean water (in relation to rural sanitation).

These points constitute the pillars of the long-term objectives for the basin as set forth in the SAGE itself. At this point, we are at the end of the second stage in this patrimonial approach. The discussion is now on its way to reaching these objectives. The negotiation process we are dealing with in this paper deals with how to reach the second objective, i.e., sharing water among users in order to observe minimum inflow in the river. The third stage has already occurred and scenarios have already been proposed with the following elements: 
- prohibition of the extension of irrigation facilities,

- finding new complementary resources to aid in satisfying water needs in the low water season,

- defining allocation rules among farmers.

Farmers are the major consumers of water for irrigation, which is mainly that of corn fields: irrigation is the main reason for water pumping, with $80 \%$ of the irrigated fields ( $3000 \mathrm{ha}$ ) being on the downstream part of the river. This explains this focus on farming scenarios for the minimum flow objective.

Farmers are partially organized within three irrigation systems managed by users' associations: three-quarters of the irrigated area falls within one of these three irrigation systems, and $85 \%$ of farmers belong to at least one of these three associations. Remaining irrigated areas are irrigated through wells in the alluvial aquifer. These associations are represented in CLE by the president of one of them, then seconded by the president of another one. Until now, whenever an emergency situation occurs, they are faced with pumping interdictions, based on the prefect's decision.

The Cemagref has been asked by the CCVD (Communauté de Communes du Val de Drôme, an association of municipalities of the basin that is hosting the secretariat of CLE) for support in defining negotiated water allocation rules among farmers in order to observe the minimum flow objective. The main goal is to reach an alternative collective solution to interdictions imposed from the top. They requested that we simulate several scenarios of the restriction rule, as well as complementary resource availability, with an emphasis on:

- water flow at downstream part of the river,

- user restrictions in water pumping,

- use of water from complementary sources.

This request is actually based on a need for support in the fourth stage of the approach: how one gets from the various scenarios proposed to a single one that will be followed by stakeholders and farmers.

Stakeholders involved have been limited to farmers in the downstream part of the river, where most pumping takes place.

The negotiation pattern faces almost no conflict or disagreement among farmers as regards water allocation rules. Instead, it demonstrates the cohesion among members of the farming sector when dealing with the CCVD and trying to re-interpret the agreement in the SAGE. Therefore, the most obvious, albeit minor, conflict takes place between the basin institution that has the SAGE on one side and stakeholders on the other side. The farm population is not that homogeneous, however: farmers with individual wells, who are not organized and thus not really represented in negotiations, are considered the "black sheep" and play the role of scapegoat.

Moreover, the whole context is evolving with:

- occurrence of droughts, causing expectations for critically dry years to evolve,

- political stakes, such as local elections, which cause new scenarios to appear and others to become taboo, 
- national and European agricultural policy, which cause interests in specific crops demanding more or less on water to evolve.

Tools used in this process had to be either adaptable or redesigned during this evolving process. Two of them have been designed successively, each one very different in form, slightly divergent in objectives for use, but used with the same protocol. Both are designed as tools for facilitators of the collective decision process: they were handled by us, but results and demonstrations were chosen and managed by CLE, whose role is to act as a facilitator in this process.

\subsection{An initial model aimed at assessing collective consequences of scenarios}

Taking into account that very little was known about farmers' behavioral patterns regarding their cropping and irrigation practices, an initial model has been designed, called SimSage. Its purpose was to assess collective consequences of various scenarios of allocation rules and complementary resource availability, in order to identify those to be explored more thoroughly. It is an operator which transforms data files of PET, upstream discharge, structural data regarding pumping capacities, irrigated areas and complementary resource flow capacities, into simulation result files for downstream flow level, water pumping restriction levels, occurrence of crises, and volume of water taken from complementary resources. Scenarios are constituted by choosing a year to simulate (data base gathers all daily data from 1971-1996), a complementary resource, and water pumping restriction rules. Table 1 below sums up the overall pattern of SimSage.

Individual pumping through wells in the alluvial aquifer make up a fourth group whose area is the sum of areas irrigated, and whose pumping capacity is the sum of individual pump capacities. Farming practices are not taken into account, the only differences being that plots belong to one irrigated system or the other. Scenarios of water pumping restrictions are described with a rate of diminution of pumping relative to climatic needs and complementary resources collected by each irrigation system. The time step is one day and each simulation covers the period from April 1st to September 30th. Field experts from the

Table 1. Structure of SimSage model

\begin{tabular}{|c|c|c|}
\hline \multicolumn{3}{|c|}{$\begin{array}{l}\text { Scenario: } \\
\text { - Reference year for hydro-climatic data } \\
\text { - Complementary resource availability } \\
\text { - Water pumping restriction rule }\end{array}$} \\
\hline \multicolumn{2}{|c|}{ Input data } & \multirow[t]{2}{*}{ Simulated results } \\
\hline Hydro-climatic & Structural & \\
\hline $\begin{array}{l}\text { - PET } \\
\text { • Upstream dischage }\end{array}$ & $\begin{array}{l}\text { - Irrigated area } \\
\text { - Pumping capacities } \\
\text { - Complementary resource capacities }\end{array}$ & $\begin{array}{l}\text { - Downstream flow level } \\
\text { - Water pumping restriction level } \\
\text { - Crisis occurrence } \\
\text { - Water taken from complementary resources }\end{array}$ \\
\hline
\end{tabular}


water agency assume that water use happens based on the following scenario: all the collectively irrigated plots are sown with corn irrigated at $70 \%$ of their needs and all individually irrigated plots are sown with corn irrigated at $87 \%$ of needs. This leads to computation of the amount of water pumped by each irrigated system and the set of individually irrigated plots, after which downstream flow is computed.

This first model has been programmed as a Macro ${ }^{\circledR}$ Excel $^{\circledR}$. It has been used through the demonstration of simulated results on graphs featuring downstream flow and water pumping restriction results series as well as evaluations of complementary resource mobilization for entire seasons and crisis events, all based on representative years. ${ }^{3}$ Figures 1 and 2 show graphs used in a meeting presenting simulations to foster negotiotion: simulated deficit in water flows on the downstream part for the given year and complementary resource scenario (Figure 1) and the occurrence of restriction rule enforcement for the given year and complementary resource (Figure 2). These demonstrations have been shown to CCVD, who then asked us to present them, first to presidents of the farming associations, then to the CLE assembly and in open sessions to which all farmers in the area were invited. In these three cases, the main objective of the CCVD and committee board of CLE was to provide an explanation of the on-going process regarding low-water management. No votes were expected. An informal agreement for a continuation of the process could be inferred.

\subsection{A second model aimed at supporting a definition of formal allocation rules}

In the second step, the dialogue among the farmers' association and the CLE committee board (mainly represented by CCVD) was based on the definition of practically enforceable allocation rules. Assumptions in the design of SimSage were incompatible with that purpose. Pumping reductions are indeed computed as a percentage of total plant needs based on PET determined by the climate. This cannot be controlled nor even clearly defined since this climatic demand may be known only ex-post. We had to go for a model with reference levels defined ex ante and known by farmers and farmer association presidents prior to the beginning of cropping season. Moreover, demonstrations of series of simulation results through Excel ${ }^{\circledast}$ graphs were difficult to understand. SimSage actually had all the makings of a black box, with the dual danger of being believed more than it should be on one hand and rejected as a whole on the other, and thus not discussed.

Therefore a second model, GibiDrome, has been designed in order to tackle these requirements. Same data files are used and the same assumption is made that corn is grown and irrigated on $100 \%$ of the whole area. Whenever a restriction might occur, maximum pumping allowance is settled according to plot area and mean climatic corn water demand at a 10 day time step.

It is an Agent-Based Model using mainly the following classes of agents ${ }^{4}$ : FarmerAssociation, Farmer, Cle, River, Crop, Plot. Architecture is described more precisely through the UML class diagram in Figure 3. The time step is again one day. For each time step, flow is generated upstream and each plot computes its water needs. Irrigation systems compute needs of all their plots and take it in the river if there is enough flow in it, but also 
simulations of water deficit at Seuil des Pues with data of year 1993

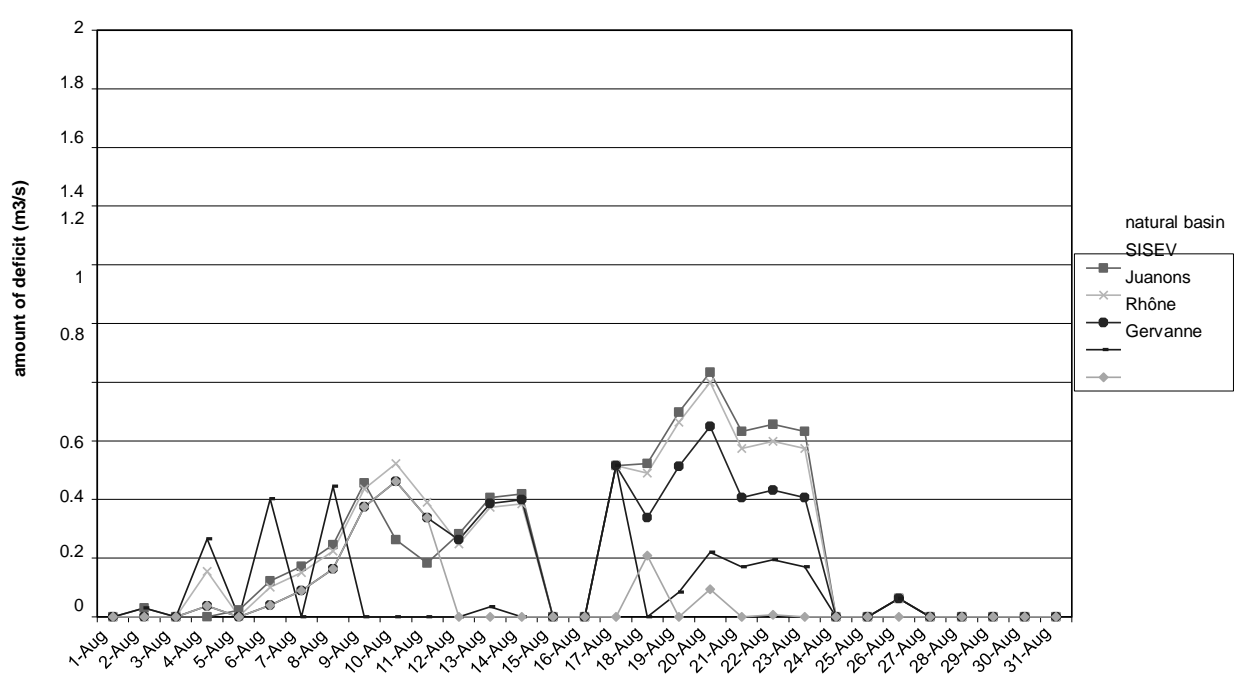

Figure 1. Deficit in water flow according to objective level at downstream part. Hydroclimatic data used here are from 1993, a medium-dry year, and simulations compare scenarios of alternative complementary resources.

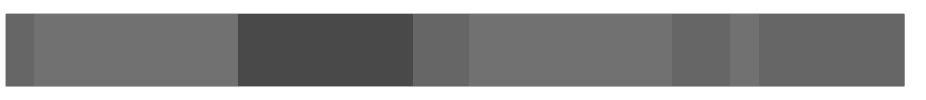

« Natural » basin

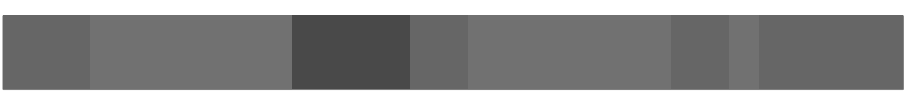

Situation actuelle

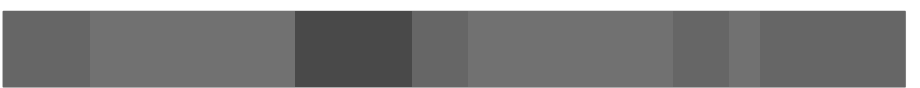

Juanons

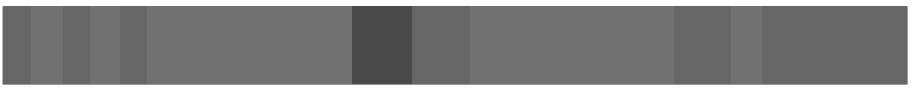

Rhône

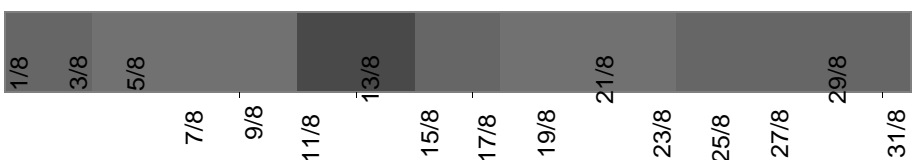

Gervanne

Normal situation

restriction $\mathrm{C} 1$

restriction $\mathrm{C} 2$

Figure 2. Enforcement of restriction levels C1 (minus 20\%) and C2 (minus 40\%). Hydroclimatic data for 1993 are used (medium-dry year) and various complementary resource scenarios are compared. 
if they have the capacity and the allowance to do so. Cle updates the possible restriction level. Farmers choose their cropping patterns for the following activities: sowing, irrigating, harvesting. The crop itself computes its yield according to the possible occurrence of water stress throughout the cropping season. Farmer constraints, such as rotation of irrigation equipment among plots on the farm have then been represented, as well as the avail-

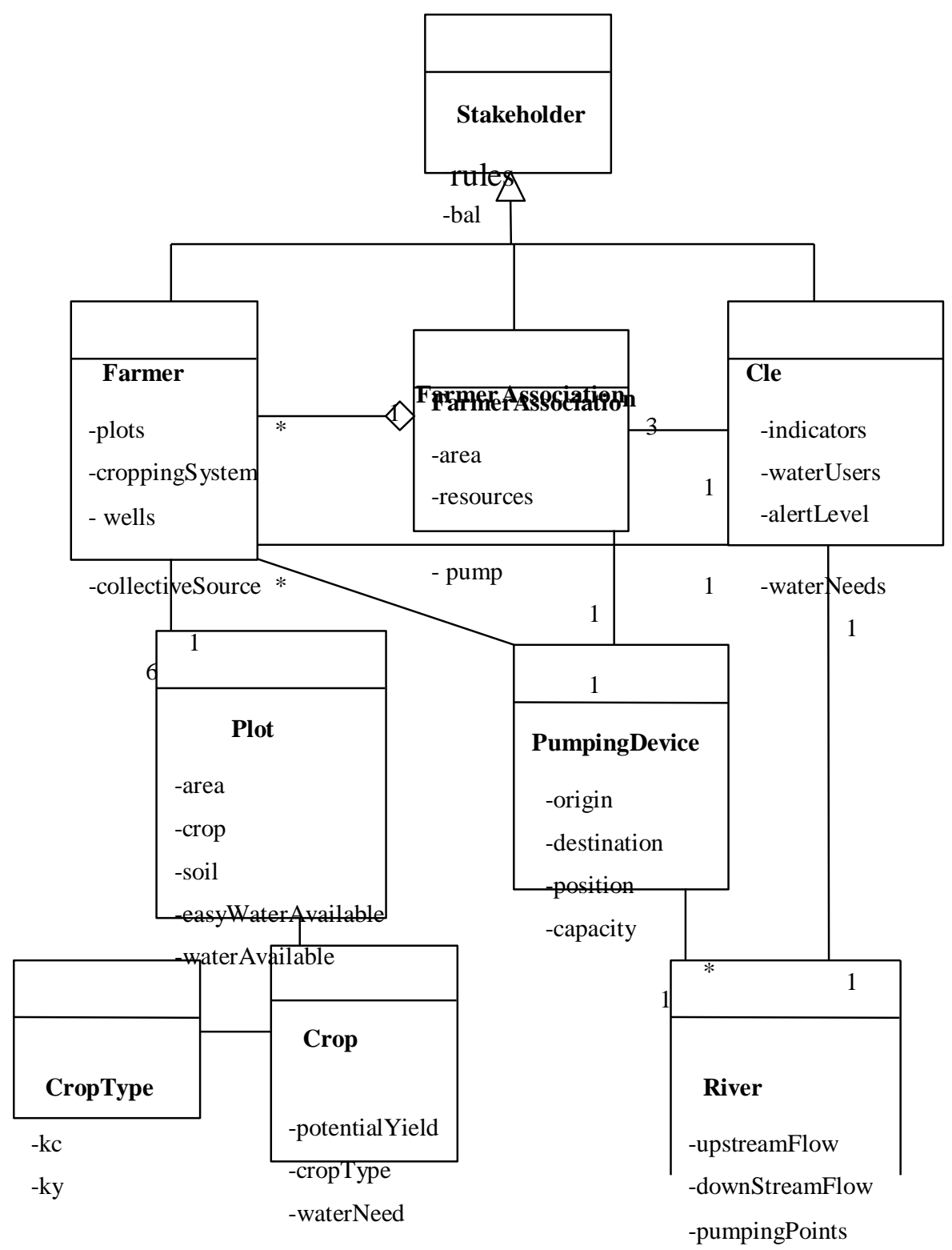

Figure 3. UML class diagram of principal entities in GibiDrome. 
ability of water at the plot level and not at the river level. Not surprisingly, this means that water has been brought from the river to the plot. Consequently, a rule for beginning irrigation has to be represented, instead of the implicit permanent irrigation assumed by models when considering water availability at the river level. From there, working-time management is tackled indirectly through this beginning irrigation. Thus, the modeling process helps to alleviate some implicit assumptions of SimSage. All these rules in the model are based on individual interviews either with farmers, leaders of water users' associations (Zanker 1999) or Cle officials.

Figure 4 below features the main interface of GibiDrome. Use of the model begins with a description of structural assumptions based upon the spatial components of the interface. The left part of the interface provides simulation management keys and particularly within the "scenarios" box, menus to choose a scenario with an item in each category (hydro-climatic data, complementary resource available, water pumping restriction rule, population of individual rules for starting irrigation season, population of individual cropping strategies, population of soil depth characteristics). These populations of individual patterns are described by percentages of farmers or plots in each type. The "Scenarios" box is looked at in order to state a value for each of the six items. For each of these, choices are deter-

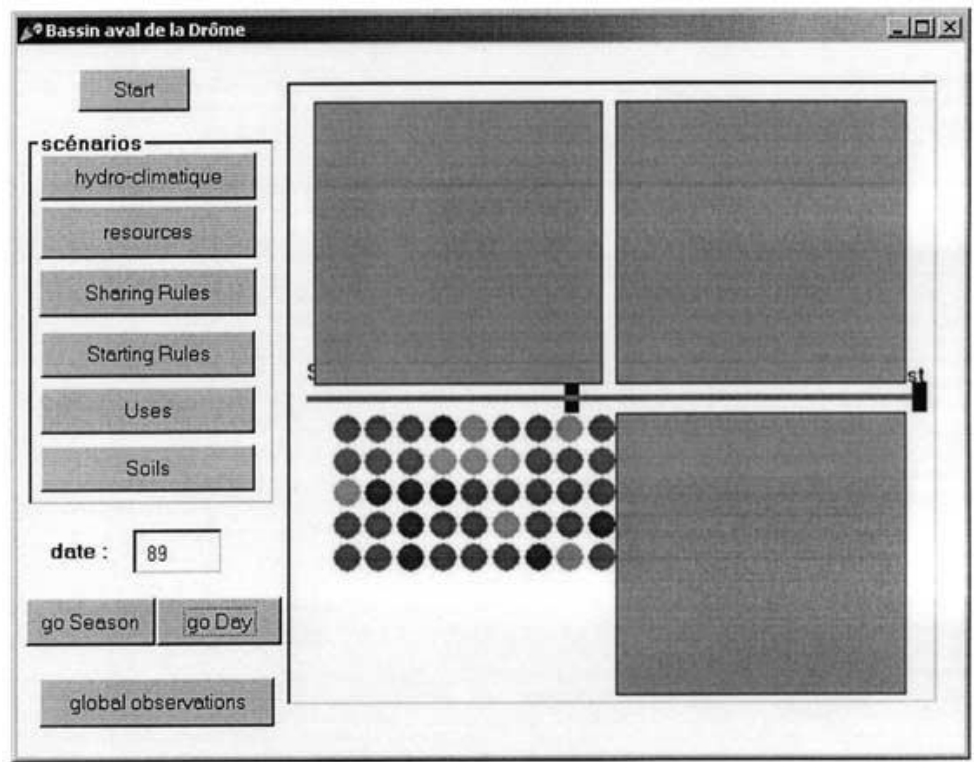

Figure 4. GibiDrome interface. 
mined for global characteristics using available data or rules and resources that are either pre-existing or anticipated, and for individual patterns such as the ratio of entities of each possible type. These patterns are defined according to available data or rules that are likewise pre-existing or anticipated. These populations are chosen by users of the model and are not constrained by the model itself, except that each Farm or Plot entity has to possess these characteristics. After choosing the scenario, simulation is run publicly with the same stakeholders who participated in the choice of a scenario.

The main window features a simplified representation of the downstream part of Drome river basin, with circles representing farms and squares representing irrigated systems, the spatial basis being for Farmer and FarmerAssociation agents respectively. The colour is related to ratio of plots in each entity suffering from water stress at current time step, the right black rectangle stands for upstream part ("pont de Crest") whereas the left one stands for "seuil des Pues" (downstream part where objective flow level is fixed). All plots and irrigated systems take water upstream from this "seuil des Pues".

Several indicators are then proposed, concerning either the global level or specific entity available through its spatial track on the interface. They concern either the evolution of possible water stress for plots of the farm or irrigated system, enforcement of the restriction rule, or downstream water flow level. Zoom can be done for each indicator on specific periods for which stakeholders want more insight. Figures 5 and 6 feature the same period with respect to the ratio of plots with water stress throughout the season on one of the irrigated systems (Figure 5) and enforcement (and level) of the restriction rule (Figure 6). These indicators may encourage discussion. The two graphs in Figures 5 and 6 are especially interesting, since it appears that water stress is due not only to enforcement of a restriction rule but also possibly to a late start to irrigation. Simulations do feature some water stress at the end of June, whereas no restriction is enforced at this time. Therefore, if the question under discussion is water stress throughout the cropping season, these stresses should be discussed as well, or the issue has to be re-framed with a view towards water stress in August, for example. In both cases, it encourages the clarification of positions and viewpoints of stakeholders in the discussion. This leads to a new analysis by stakeholders with whom the model is used, as well as by the model designer. The focus in discussions on enforcement of restriction rules becomes less relevant in view of these new pieces of information. Domination of the physical and institutional possibility of access to water in the representation of constraints to farming must be shared with the farmer's ability to take water. This is discussed more thoroughly in the following section. Support to the collective decision process is thus achieved through a re-framing of the discussion and evolution of the representation of the system by stakeholders rather than by providing specific agreements.

GibiDrome is an evolving model and was designed to accept new scenarios of complementary resources, since the requirements with these scenarios are evolving with the project itself. For example, differentiation of plots according to soil water capacity was introduced following the first demonstrations since stakeholders requested it as a major factor needed to understand and analyze the whole system. There has been an incremental set of demonstrations first to Cle officials and later to presidents of water users' associations and a few individual farmers' representatives. It has fostered a common learning process. This in turn 


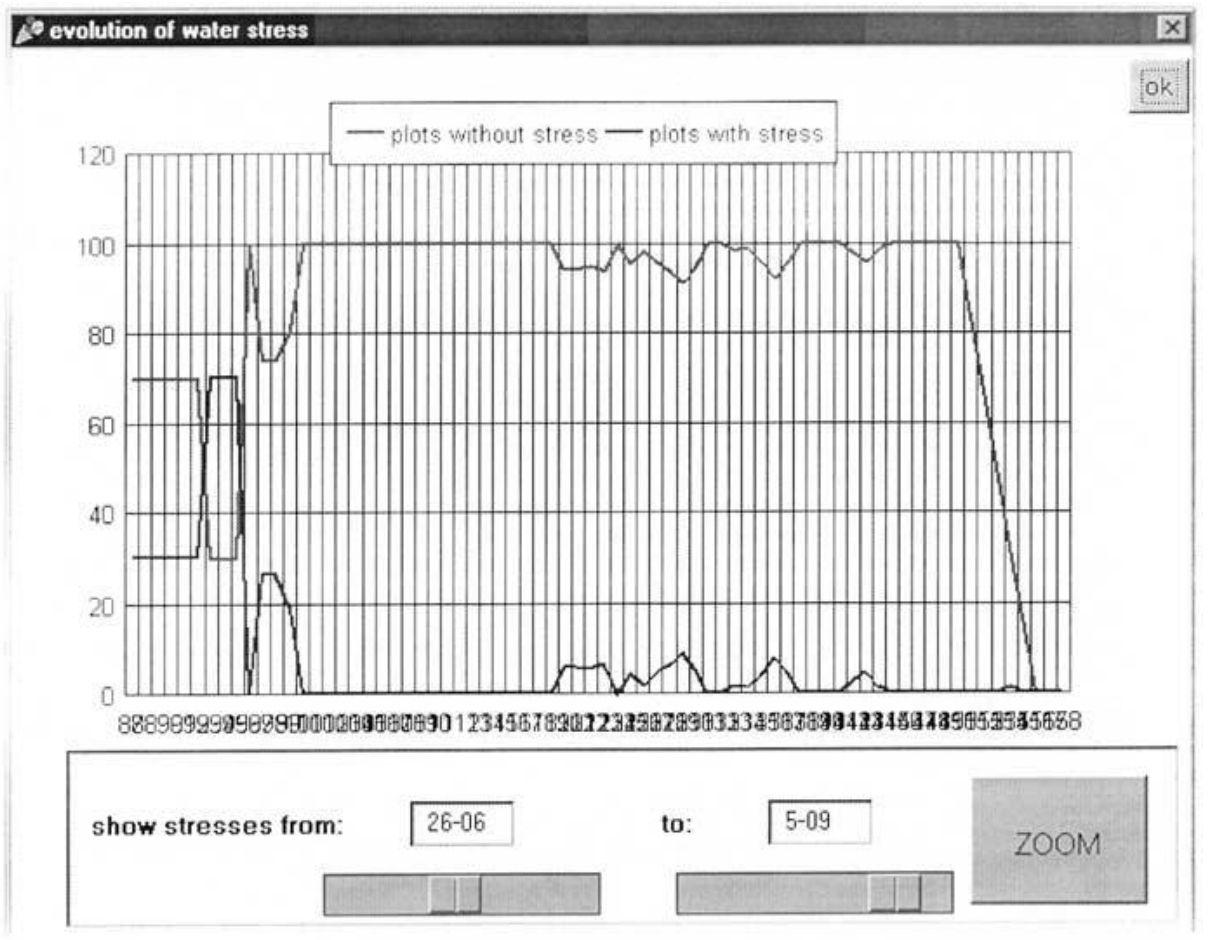

Figure 5. Ratio of plots with water stress during period from June 28th to September 6th, for the most downstream part of irrigation system, with blue line indicating ratio of plots with stress, whereas red line indicates ratio of plots without any stress.

encourages these stakeholders to open discussions on previously unrevealed practices (cropping patterns), and to discuss water allocation and use across the cropping patterns.

\section{Contributions of MAS as a negotiation support tool}

Even though both tools have not been used with the same objective and at the same time, this experiment lets us draw some preliminary conclusions regarding the contributions of MAS in a negotiation process as a support tool in comparison with other, more classical, modeling tools.

A strict comparison between both tools is not possible, since it would involve control of many parameters, which, by their nature, are not controllable. The usual "everything being equal" cannot be applied in an action research program. It is therefore more useful to acquire a more in-depth understanding of processes involved during interaction between tools and stakeholders than to undergo a thorough comparison. 


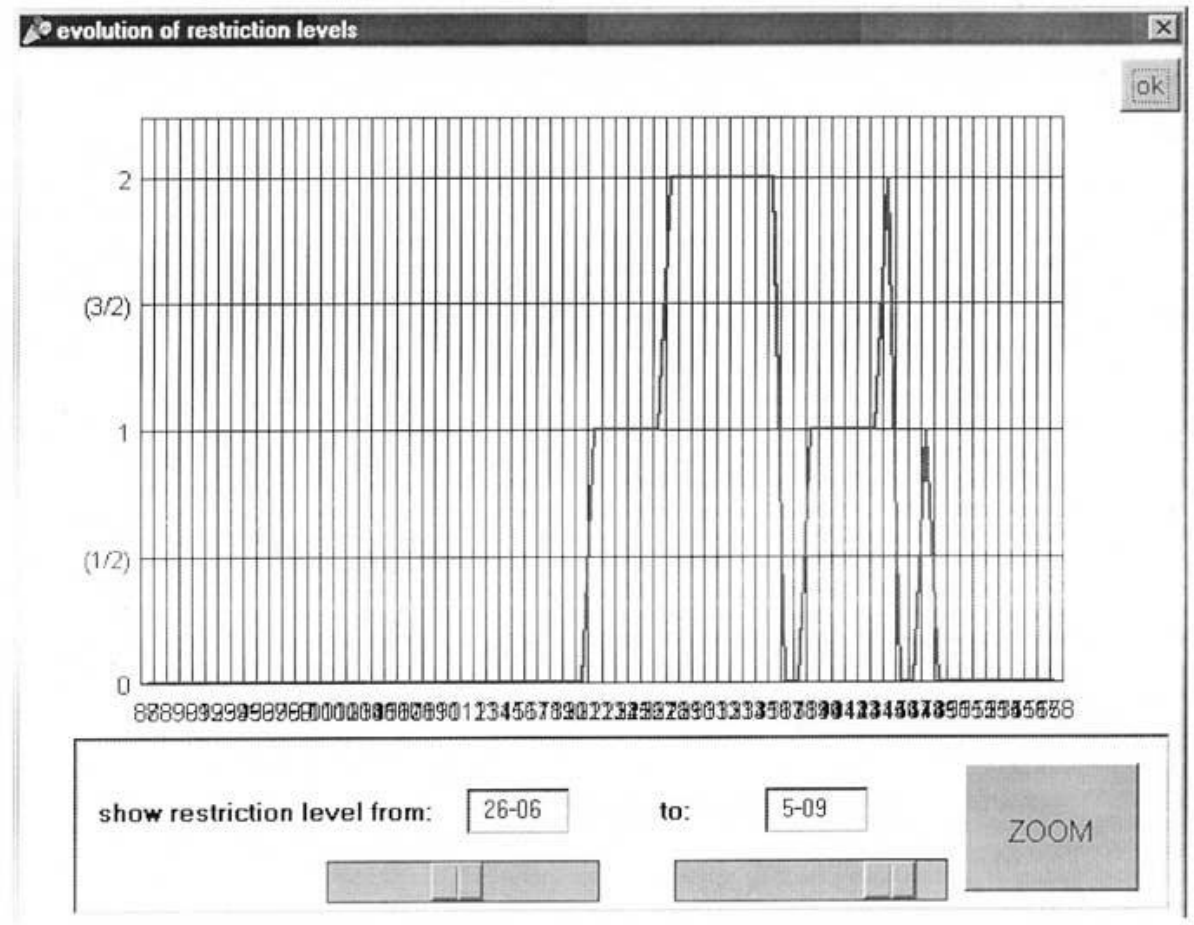

Figure 6. Enforcement of restriction rule by Cle for period from June 28th to September 6th, with level 1 corresponding to a $20 \%$ restriction and level 2 to a $40 \%$ restriction.

A few conclusions may be linked to requirements previously stated for models to be used in support of a patrimonial approach for complex systems. We will not touch on the ability of MAS to serve as a cross-disciplinary tool, which has already been shown, for example in (Barreteau and Bousquet 2000; Bousquet et al. 1999; Gilbert and Troitzeh 1999). This discussion will concentrate on two other features: adaptability of the tool for designers and its capacity to bring new suitable and legitimate information for stakeholders.

\subsection{Following evolutions of context}

Negotiation processes such as water allocation happen in open systems and may thus take place in a highly evolving context. A set model has to be written each time in order to fit a specific context. This is the case for the Drôme River Valley: From one year to another, scenarios to be considered, agreements among stakeholders, cropping preferences, irrigation equipment, and drought risk perception evolve in relation to the seasons, political stakes at local elections, and market and agricultural policies. With SimSage, each evolution in scenario a of complementary resource, for example, requires a thorough verification of the 
whole model for its compatibility, as well as a lot of rewriting. However, with GibiDrome, there is a way to add a new resource that did not yet exist during design.

The flexibility of object programming on which Agent-Based Modeling is based is a first feature that favors this evolutionism. However, we are also involved in a companion modeling process here, (Bousquet et al. 1999, 2002) in which the decision pace can be quite rapid $^{5}$. As is the case with other modeling methodologies such as Evolutionary System Design (Hämäläinen et al. 2001; Shakun 1996) this companion modeling is based on a cycle revolving with field study, modeling activity, simulation analysis and feed-back to the field. However, it is also based on a strong interactive pattern: interaction with the decision process takes place in various stages at each cycle. With agent-based Modeling comes the possibility of having a modeling pace that is shorter and thus adaptable to local dynamics, whereas the cycle is driving the continuity of subsequent versions of models. Being more flexible, it may be adapted almost on demand, as was done by Patrick d'Aquino in Senegal (d'Aquino et al. 2001; Lynam et al. 2002), with a good tracing between various stages of the model. In his case, he went through a real model designed by stakeholders themselves.

GibiDrome has led to negotiation of some specific features of models, such as actual water consumption to be assumed in the model, or soil characteristics. This negotiation of a model is also a way to foster discussion as to why it is important that the collective decision process produce a result. Realism of the model's content is less at stake than the strategic consideration of choosing one value or another. In this specific case of specifying assumptions of actual water consumption in GibiDrome, it is quite easy to assess the consequences on model outcomes based on one choice or another. Therefore, discussion has to focus on reasons for negotiation. Here the alternative is between the crisis declared by the prefect, with specific interdictions, and the design of collective rules making it possible to prevent a crisis from occurring: some overly unrealistic values would lead to an inefficient tool, and thus the status quo. The choice between status quo and dialogue is then put back on the discussion table.

When used for negotiation support purposes, companion modeling is comparable to other negotiation support methodologies, such as Single Negotiation Text (Fisher and Ury 1981). Here the cycling companion modeling process actually provides a kind of "Single Negotiation Model". The main difference is that rewriting does not necessarily concern only the facilitator but might also concern the stakeholders. A single model is provided to the negotiation arena at each loop of the process.

\subsection{MAS as a reframing tool for negotiation process}

Besides its capacity for adaptability, making models continually relevant to issues at stake in a dialogue, MAS also brings new viewpoints to stakeholders. Driven by the need to specify behavioral patterns of agents such as farmers in order to obtain the dynamics of water use, we had to simulate cropping and irrigation practices for each farmer, such as those practiced at the beginning of irrigation in particular. Occurrences of water stress appear to be very sensitive to this factor, as has already been shown in Figure 5. This has caused the CLE to wonder about that factor in the real world and to seek more information. It also 
opens the field of discussion as we have done here with regard to constraints for early starting of irrigation. This has resulted in more discussion on constraints and also on each player's objectives. The objectives of farmers not only limit events of water stress, but also limit constraints in cropping and irrigation activities at key moments in the cropping season. In this case, use of a MAS is a means to making negotiation go from a zero-sum game to a win-win situation.

Communications among agents in the model actually overflow in the real world of the negotiation: when answers are missing due to a misconception on the part of the message receiver, the model designer is induced to explore in greater depth the real behavioral patterns of entities represented by the receiver agent in trouble. The model designer followed by the facilitator and eventually the stakeholders are then asked whether or not this deficiency in design of agents corresponds to a really important feature, which must then be discussed. New features in the model thus appear. These may correspond to taboos or topics perceived as outside the scope of the case study. The starting date for irrigation is currently not discussed: all expectations for yield improvement are based on water pumping during the season. However, such water stress due to late beginning of irrigation has already been observed (Labbé et al. 2001) in other areas. However, it is mainly due to constraints within the farm's operation as regards work availability from farmers.

Model designers and facilitators are also taking advantage of MAS to acquire new information. So MAS design encourages stakeholders to provide more information on their behavioral patterns, or at least on the image they want to present. For example, leaders of Irrigated Systems Associations specified some diversity in cropping patterns in order to feel better represented. Discussion of the model's assumptions is thus complementary to more traditional interviewing techniques.

The design of a MAS requires an exhaustive elaboration of relations among entities. Any assumptions regarding the diffusion of information among agents in the model must be made explicit. For example, the design of GibiDrome raised the issue of knowledge of restriction rule enforcement by individual farmers. In SimSage, it is implicit that they would automatically know and conform to it. However, in GibiDrome, Farmer agents consult the Cle agent regarding the actual state of the restriction rule, and in a second stage they process this information to adapt their water pumping behavior accordingly.

Therefore, it is a first step towards opening the black box of a model. The separation of information and deliberation processes as well as the specification of interactions among agents make clearer, at least for the model designer, the many implicit assumptions of models, decreasing its black box aspect. This clarification at the model design level paves the way for clarification at the model communication level through the providing of elements for explaining model content. This takes the direction of favoring discussion on the model, but is not enough in itself. In first uses of GibiDrome with small groups of water users' association leaders, content has been explained through a narrative on the basis of the various entities appearing in the interface, as well as on the elements of choice for scenarios. It seems it has been sufficient. However, in other cases, some other tools such as role-playing games, dedicated to the communication of model content need to be designed, (Barreteau, Bousquet, and Attonaty 2001; d' Aquino et al. 2002). 


\section{Conclusion}

This experiment in the Drôme River Valley with the SAGE water allocation collective decision process shows three major benefits of the use of Agent-Based Models as negotiation support tools:

- they keep negotiation support tools relevant to negotiation stakes through adaptation to context evolution,

- they enlarge the field of information of stakeholders through multi-level viewpoints on the system dynamics,

- They reveal interconnected topics in the discussion that were previously kept outside of scope of the discussion, whether consciously or not.

Agent-Based Models are thus efficient in supporting negotiation. This is shown here in a specific context: the facilitation point of view and organization of the whole collective decision process as a patrimonial approach. Their use in other contexts is at stake in several on-going works.

Their protocol of use should be considered carefully because they might strongly reinforce information asymmetry. Here CLE is the client of the research program and thus of the modeling. Information on the simulations is first communicated to them, and it could be tempting for them to select some scenarios first.

\section{Acknowledgements}

We are grateful to CCVD (Communauté de Communes du Val de Drôme) and the French Ministry of the Environment's program called "Concertation et décision en environnement" (dialog and decisions on the environment) for their support in providing data and financing this research. We also wish to thank our colleagues from the Cirad and INRA for the rich discussions on companion modeling, Multi-Agent Systems and patrimonial approach.

\section{Notes}

1. France is divided into six water agencies, whose role is to manage water and organize stakeholders at great basin scale. Each agency has a territory based on one of the major French river basins and the neighboring coastal rivers.

2. In France, local state authority representative.

3. The 26 years have been separated into 4 quartiles according to their dryness, and the most recent year of each quartile has been taken as a representative year, with the assumption that stakeholders will remember this year better and thus have a greater appreciation for the simulation results.

4. Here, Agent is defined relative to computer science. It is an autonomous entity, situated in an environment and interacting with other agents and its environment. Agent-based modeling uses these entities to represent entities from the real world, such as actors for example, but also according to the requirements of the representation tasks, water bullets, associations, sand grain, etc. In this paper, "agent" always refers to the modeling world, while "actor" is preferred when dealing with the real world.

5. It is actually highly variable but may be very rapid at some moments. 


\section{References}

Babin, D. and A. Bertrand. (1998). "Managing Pluralism: Subsidiarity and Patrimonial Mediation," Unasylva $49,19-25$.

Barreteau, O. and F. Bousquet. (2000). "SHADOC: A Multi-Agent Model to Tackle Viability of Irrigated Systems," Annals of Operations Research 94, 139-162.

Barreteau, O., F. Bousquet, and J.-M. Attonaty. (2001). "Role-Playing Games for Opening the Black Box of MultiAgent Systems: Method and Teachings of its Application to Senegal River Valley Irrigated Systems," Journal of Artificial Societies and Social Simulations 4 (http://jasss.soc.surrey.ac.uk/4/2/5.html).

Benbasat, I. and J. Lim. (2000). "Information Technology Support for Debiasing Group Judgments: An Empirical Evaluation," Organizational Behavior and Human Decision Processes 83, 167-183.

Bousquet, F. et al. (1999). "An Environmental Modeling Approach. The Use of Multi-Agent Simulations," in F. Blasco and A. Weill (eds.), Advances in Environmental and Ecological Modeling. Elsevier.

Bousquet, F. et al. (2002). "Multi-Agent Systems and Role Games: An Approach for EcoSystem Co-Management," in M. Janssen (ed.), Complexity and Ecosystem Management: The Theory and Practice of Multi-agent Approaches. Edward Elgar Publishers.

Burkardt, N., B. L. Lamb, and J. G. Taylor. (1998). "Desire to Bargain and Negotiation Success: Lessons About the Need to Negotiate from Six Hydropower Disputes," Environmental Management 22, 877-886.

d'Aquino, P. et al. (2002). "The Role Playing Games in an ABM Participatory Modeling Process: Outcomes from Six Different Experiments Carried Out in the Last Five Years," in Proceedings of ISEE 2002, Sousse, Tunisia.

d'Aquino, P. et al. (2001). “Jeux de rôles et simulations multi-agents," in E. Malézieux, G. Trébuil, and M. Jaeger (eds.), Modélisation des agro-écosystèmes et aide à la décision. Cirad-Inra, Paris, France.

Doran, J. E. (1999). "Trajectories to Complexity in Artificial Societies: Rationality, Belief and Emotions," in T. Kohler and G. Gummerman (eds.), Dynamics in Human and Primate Societies. Oxford University Press.

Driessen, P. P. J., P. Glasbergen, and C. Verdaas. (2001). "Interactive Policy Making - A Model of Management for Public Works," European Journal of Operational Research 128, 322-337.

Druckman, D., R. Harris, and B. Ramberg. (2002). "Computer-Assisted International Negotiation: A Tool for Research and Practice," Group Decision and Negotiation 11, 231-256.

Faratin, P. et al. (2001). "Simple Negotiating Agents in Complex Games: Emergent Equilibria and Dominance of Strategies," Seattle, USA, pp. 42-53, in Proceedings of 8th Int Workshop on Agent Theories, Architectures and Languages (ATAL-01), Seattle (USA).

Faure, G. O. et al. (eds.). (1998). La négociation - situations et problématiques. Nathan.

Ferber, J. (1999). Multi-Agent Systems. Reading: Addison-Wesley Longman.

Fisher, R. and W. Ury. (1981). Getting to Yes: Negotiating Agreements Without Giving In. Boston: HoughtonMiffin.

Funtowicz, S. O. et al. (1999). "Information Tools for Environmental Policy Under Conditions of Complexity," European Environment Agency 9.

Gilbert, N. and K. Troitzeh (1999). Simulation for the Social Scientist. Open University Press.

Green, K. C. (2002). "Forecasting Decisions in Conflict Situations: A Comparison of Game Theory, Role-Playing and Unaided Judgment," International Journal of Forecasting, forthcoming.

Grosz, B. J. (2000). "What's Needed to Build Team Players," in Proceedings of ICMAS 2000, Boston.

Hämäläinen, R. P. et al. (2001). "Evaluating a Framework for Multi-Stakeholder Decision Support in Water Resources Management," Group Decision and Negotiation 10, 331-353.

Labbé, F. et al. (2001). "Modeling Irrigation Scheduling to Analyze Water Management at Farm Level, During Water Shortages," European Journal of Agronomy 12, 55-67.

Lim, J. (2000). “An Experimental Investigation of the Impact of NSS and Proximity on Negotiation Outcomes," Behavior and Information Technology 19, 329-338.

Lynam, T. et al. (2002). "Adapting Science to Adaptive Managers - Spidergrams, Belief Models and Multi-Agent Systems Modeling," Conservation Ecology 5 (http://www.consecol.org/vol5/iss2/art24).

Moss, S. and P. Davidsson (eds.). (2001). Multi-Agent-Based Simulations. Springer.

Ostrom, E. (1990). Governing the Commons: The Evolutions of Institutions for Collective Action. Cambridge University Press. 
Rouchier, J. et al. (1998). "Evolution and Co-Evolution of Individuals and Groups in Environment,” in Proceedings of ICMAS '98, Paris.

Shakun, M. E. (1996). "Modeling and Supporting Task-Oriented Group Processes: Purposeful Complex Adaptive Systems and Evolutionary Systems Design," Group Decision and Negotiation 5, 305-317.

Simonovic, S. P. (2000). “Tools for Water Management, One View of the Future,” Water International 25, 7688.

Teulier-Bourgine, R. (1997). “Les représentations: médiation de l'action stratégique,” in M.-J. Avenier (ed.), La stratégie chemin-faisant. Economica.

Weber, J. (1996). "Conservation, développement et coordination: peut-on gérer biologiquement le social?" in Proceedings of Colloque Panafricain Gestion Communautaire des ressources renouvelables et développement durable, Harare.

Weber, J. (1998). "Perspectives de gestion patrimoniale des ressources renouvelables," in P. Lavigne Delville (ed.), Quelle politique foncière en Afrique rurale? Karthala-Coopération française.

Zanker, S. (1999). Modélisation et gestion de la demande agricole en eau dans la Drôme, cas de la mise en application du SAGE Drôme. Cemagref 
